\title{
Estudo de Tendência de Variáveis Aplicado a Plataforma Data-Driven em uma Produção Industrial *
}

\author{
Vinicius Martins Duarte* Claudio Henrique Tosin ${ }^{* *}$ \\ Gilberto Reynoso-Meza*** \\ * Programa de Pós-Graduação em Engenharia de Produção e Sistemas, \\ Pontifícia Universidade Católica do Paraná, PR, (e-mail: \\ martins.duarte@pucpr.edu.br). \\ ** Autor Autônomo, PR, (e-mail: claudiotosin@yahoo.com.br). \\ *** Programa de Pós-Graduação em Engenharia de Produção e \\ Sistemas, Pontifícia Universidade Católica do Paraná, PR, (e-mail: \\ g.reynosomeza@pucpr.br).
}

\begin{abstract}
This paper aims to study the application of the multiple linear regression technique for a trending study of some variables in a data driven platform. The purpose of this application is based on the improvement of controlling an industrial process. The variables studied were the values relatives of the gap between products in the production line and the deviations related to this gap, in order to increase the productive flow and reduce the variation of the process respectively. For the gap value the applied technique presented some significant numerical results, but for the deviation did not have the same behavior.

Resumo: Este artigo tem como objetivo estudar a aplicação da técnica de regressão linear múltipla para um estudo de tendência de variáveis em uma plataforma de dados dirigidos. O objetivo dessa aplicação se fundamenta na melhoria de controle em um processo industrial. As variáveis estudadas foram os valores de espaçamento entre produtos na linha de produção e os desvios relativos à esse espaçamento, com o intuito de aumentar o fluxo produtivo e reduzir a variação do processo respectivamente. Para o valor de espaçamento a técnica aplicada apresentou resultados numéricos significativos, porém para o desvio não teve o mesmo comportamento.
\end{abstract}

Keywords: Multiple Regression; Industry 4.0; Lean Manufacturing; Data-Driven; Industrial Process.

Palavras-chaves: Regressão Múltipla; Indústria 4.0; Lean Manufacturing; Data-Driven; Processo Industrial.

\section{INTRODUÇÃO}

Com o avanço tecnológico e a necessidade de melhorar os processos produtivos surgem novos desafios na produção industrial como por exemplo a automação inteligente. Segundo Tessarini and Saltorato (2018) o mundo está em uma revolução na produção onde se une os conceitos analíticos, comunicação e máquinas. Essa revolução é conhecida como Indústria 4.0. Porém, em termos metodológicos, predomina-se nas produções científicas, o estudo do tipo teórico, o que reflete a urgência de pesquisas empíricas e aplicadas diretamente em campo. Atualmente as empresas tem investido cada vez mais na coleta, análise e na aplicação de ferramentas computacionais para adquirir uma vantagem competitiva no mercado. Segundo Provost and Fawcett (2013) uma das ferramentas mais utilizadas para tomadas de decisão automáticas por meio de métodos

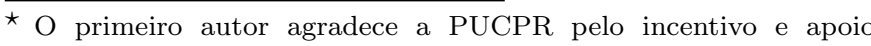
financeiro no desenvolvimento do projeto de pesquisa de mestrado Este trabalho for apoiado parcialmente pelo CNPq através das subvenções dos projetos PQ2/304066/2016-8 e UNI/437105/2018-0 computacionais é o Data-Driven, pois permite trabalhar com grandes quantidades de dados de maneira organizada.

De acordo com Lopes and Frota (2015) um dos objetivos da produção industrial moderna visa à eliminação das perdas em processos. A metodologia aplicada na indústria que visa essa eliminação de perdas é conhecida como Lean Manufacturing. A eliminação de perdas torna os processos menos custosos e mais eficazes, trazendo benefícios para os fabricantes. Para isso é necessária a utilização de ferramentas de controle estatístico. Para Rosário et al. (2015) a redução na variação de processos é um dos objetivos nos controles industriais, com o intuito de evitar perdas.

Além da redução nas perdas devido a variações nos processos, um outro objetivo na indústria é o aumento do fluxo produtivo, ou seja, de acordo com Moraes and Santoro (2006) reduzir o tempo de processos em pontos críticos conhecidos popularmente como "gargalos". De acordo com Moreira (2017), a maior parte das referências que tratam do assunto de administração da produção apresenta o conceito de capacidade, ou quantidade de produção por 
período de tempo como uma medida de eficiência da produção.

\section{ABORDAGEM E FERRAMENTAS UTILIZADAS}

\subsection{Regressão Múltipla}

De acordo com Montgomery and Runger (2013) muitas aplicações de análise de regressão envolvem situações onde há mais de um regressor, ou seja, o efeito de duas ou mais variáveis em função de uma variável objetivo. Quando um modelo de regressão envolve mais de um regressor, o mesmo pode ser denominado como modelo regressor múltiplo. Esse modelo é representado pela equação:

$$
y=\beta_{0}+\beta_{1} x_{1}+\beta_{2} x_{2}+\cdots \beta_{k} x_{k}+\epsilon
$$

onde $y$ representa a variável objetivo e os betas correspondem aos coeficientes angulares de (1), $x$ representa as variáveis que compõe o modelo regressivo e $\epsilon$ corresponde ao erro do modelo.

\subsection{Indicadores de Ajuste de Curva}

De acordo com Robinson (2003) o erro percentual de um experimento pode ser calculado pela expressão:

$$
\operatorname{Erro}(\%)=\frac{\mathrm{VA}-\mathrm{VE}}{\mathrm{VE}} \times 100 \%
$$

Onde $V A$ corresponde ao valor estimado pelo regressor e $V E$ ao valor exato da medição.

Esse indicador mostra o quanto de desvio a curva possui em relação aos dados obtidos experimentalmente.

Uma possível forma de avaliar a qualidade do ajuste de um modelo é através do coeficiente de determinação. Este indicador mostra quanto um modelo é capaz de explicar os dados coletados. O coeficiente de determinação pode ser calculado pela expressão:

$$
R^{2}=\frac{\hat{\beta}_{1} \sum_{i=1}^{n}\left(x_{i}-\bar{x}\right) Y_{i}}{\sum_{i=1}^{n}\left(Y_{i}-\bar{Y}\right)^{2}}
$$

Segundo Montgomery and Runger (2013), o valor de $R^{2}$ está contido em um intervalo entre 0 e 1 e quanto mais próximo de 1 estiver, melhor.

Outra maneira de se ter um indicador de qualidade para um ajuste de curva é pelo Erro Quadrático Médio (EQM) o qual pode ser calculado pela expressão

$$
E Q M=\frac{1}{N} \sum_{i=1}^{N}\left(x_{i k}-x_{i}\right)^{2}
$$

Nessa expressão calcula-se a diferença elevada ao quadrado entre o valor esperado e o valor da amostragem dividido pela amostragem.(Hallak and Pereira Filho, 2011)

\section{DESENVOLVIMENTO}

\subsection{Considerações Iniciais}

O presente trabalho tem como objetivo estudar uma tendência de comportamento de uma máquina industrial a respeito de dois ajustes em sua operação. Esse estudo compara diferentes curvas obtidas pela aplicação de uma ferramenta estatística. Essa abordagem é diferente de uma abordagem matemática mais completa sobre o comportamento da máquina e possui a finalidade prática de descobrir ajustes que resultam em um melhor desempenho na produção. Essa abordagem justifica uma aplicação mais simples, porém, prática do experimento que servem como base para estudos futuros com o uso de ferramentas mais complexas como por exemplo modelagens dinâmicas e aprendizado de máquina.

Vale ressaltar que este trabalho representa o início de um estudo, que resultará em um projeto que está em andamento, de uma aplicação online de análise de dados baseado em uma plataforma computacional. De acordo com a Figura 1, este trabalho estaria se referenciando a etapa de estudo das ferramentas de aproximação mais simples. Essa plataforma terá a finalidade de auxiliar o trabalho dos operadores da máquina de forma a otimizar a produção. As etapas futuras do estudo e do projeto podem ser visualizadas na Figura 1.

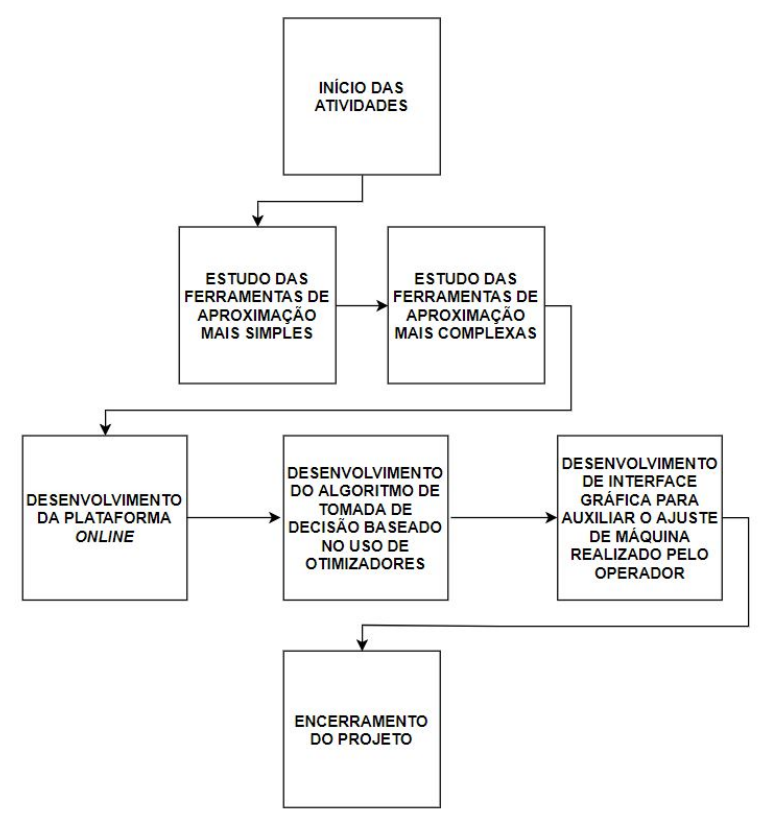

Figura 1. Fluxograma contendo as etapas futuras do estudo e projeto

Quanto a realização do experimento, a mesma consistiu em coletar dados medidos por um sensor digital de posição para cada ajuste realizado em uma máquina. Em seguida esses dados foram recebidos pelo controlador por meio de sinais digitais. Após essa aquisição de sinais, o controlador realiza operações internamente no equipamento com a finalidade de fornecer os valores númericos das grandezas de interesse para um sistema supervisório. Esse sistema apresenta a possibilidade de tabular os valores médios das medições a cada segundo para que em seguida seja aplicada a ferramenta estatística de interesse. A Figura 2 mostra o fluxograma das etapas relativas ao experimento. 


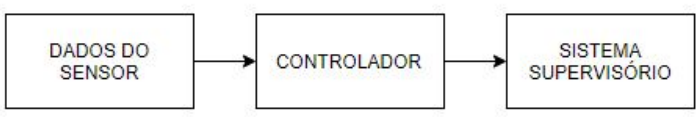

Figura 2. Fluxograma com as etapas da aquisição de dados do experimento

\subsection{A Máquina e as Variáveis de Interesse}

A máquina que está sendo estudada neste trabalho é uma do tipo alimentadora de processos e os elementos ajustáveis (destacados) podem ser visualizados pela Figura 3. Os processos seguintes correspondem a dobra e selagem do produto final. Os ajustes efetuados na máquina alimentadora implicam em uma variação no fluxo produtivo, além de reduzir as paradas de máquina por colisões entre produtos, ou seja, quando um produto se choca com outro na linha de produção. Esse fenômeno de colisão gera desperdício de material. Para manipular a variável de espaçamento entre produtos foi realizado um estudo pelo fabricante e o mesmo constatou que existem dois ajustes mecânicos externos que poderiam ser realizados pelos operadores de forma a otimizar o controle nesse ponto de processo. O primeiro ajuste externo que pode ser realizado pelos operadores da máquina é girar uma manivela para ajustar o posicionamento da máquina alimentadora em relação ao ponto sucessor dessa etapa do processo. Outro possível ajuste a ser realizado pelos operadores da máquina é relativo à altura de guias que separam os produtos durante o processo, com o objetivo de garantir que os produtos escoem respectivamente pela máquina alimentadora.

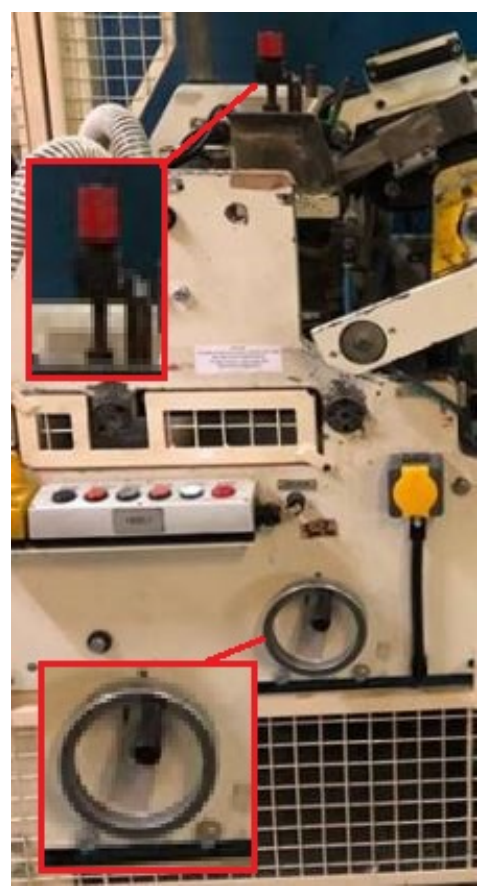

Figura 3. Máquina alimentadora

As variáveis de interesse neste estudo foram o espaçamento entre os produtos no processo em linha e sua variação. Para o fluxo produtivo a utilização do espaçamento entre produtos como variável é justificável pois o mesmo demonstra a capacidade de produção. A variação também foi levada em conta, pois é interessante para o fabricante manter um controle na estabilidade do processo e reduzir perdas que possam ocorrer quando por exemplo, dois produtos seguidos colidem durante o percurso programado. Resultando em paradas no processo que influenciam diretamente nos resultados de produção da empresa devido ao tempo que deixa de produzir.

\subsection{Aplicação da regressão múltipla}

A regressão linear múltipla tem como finalidade gerar um polinômio de tendência de comportamento de uma variável objetivo e com a utilização dessa ferramenta pode-se experimentar polinômios que possuam uma ordem maior do que apenas diretamente com a variação dos ajustes realizados, pois existe a possibilidade que de alguma maneira uma variável possa interferir na outra mesmo que indiretamente. Como para uma possível aplicação prática de um início de estudos sobre uma variável é tentar utilizar modelos mais simples, foram analisados seis comportamentos diferentes referentes as equações (4), (5), (6), (7), (8) e (9). Essas equações foram determinadas de maneira arbitrátia para que o polinômio gerado não tivesse uma complexidade muito alta, com a finalidade de simplificar um possível controle.

$$
\begin{aligned}
& y_{1}=\beta_{1} x_{1}+\beta_{2} x_{2} \\
& y_{2}=\beta_{1} x_{1}+\beta_{2} x_{2}+\beta_{1,2} x_{1} x_{2} \\
& y_{3}=\left(\beta_{1} x_{1}+\beta_{2} x_{2}\right)^{2} \\
& y_{4}=\left(\beta_{1} x_{1}+\beta_{2} x_{2}\right)^{3} \\
& y_{5}=\left(\beta_{1} x_{1}+\beta_{2} x_{2}\right)^{4} \\
& y_{6}=\left(\beta_{1} x_{1}+\beta_{2} x_{2}\right)^{5}
\end{aligned}
$$

Para fins de simplificação, os dados foram normalizados e isso reduziu o efeito do coeficiente linear da curva, resultando na exclusão do termo no equacionamento. A estratégia de interesse para o processo está no estudo da tendência do valor médio do espaçamento entre embalagens e na sua variação, para isso foram aplicadas as regressões múltiplas referentes aos polinômios descritos nas equações (2) a (7) para a determinação de duas funções objetivo, a do valor médio e a do desvio desse espaçamento.

Foi realizado um teste com uma série de ajustes tanto na manivela quanto na guia da máquina alimentadora. Para cada ajuste foram coletados dois minutos de amostra, ou seja, se a cada segundo equivale a uma amostra, dois minutos equivalem a cento e vinte amostras para cada ajuste, e com esse conjunto de dados foi aplicada a regressão linear múltipla.

Como modelos muito complexos podem trazer complicações práticas na aplicação real de melhorias em geral, a ordem máxima do polinômio foi definida como equivalente à cinco. A primeira função a ser estimada pela regressão múltipla foi relaciona a magnitude do espaçamento entre as embalagens da máquina alimentadora. Para estimar esse comportamento aplicou-se diretamente a função mvregress (MathWorks, 2006) com os valores de ajustes indicados e 
com isso obteve-se o seguinte resultado expressado pela Figura 4 onde mostra a curva em relação aos dados reais e as curvas geradas pelos polinômios de tendência.

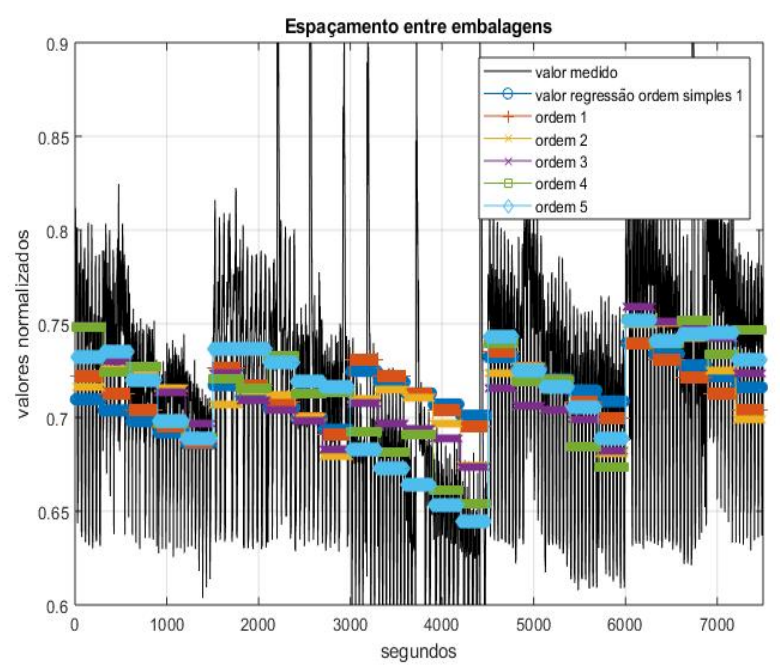

Figura 4. Espaçamento entre embalagens e os modelos regressores

Para a segunda função teve-se de utilizar uma lógica para conseguir mensurar o quanto teve-se de variação no processo. A lógica consiste em obter o valor absoluto das diferenças entre os valores de magnitude e os valores médios de cada ajuste (8). Após esse procedimento, foi gerado um novo conjunto de dados e aplicou-se novamente a função mvregress para obter os mesmos resultados relativos ao procedimento anterior, porém para a função do desvio. Os valores calculados de desvio do espaçamento e seus regressores podem ser visualizados na Figura 5.

$$
\operatorname{Desvio}(t)=\sqrt{\left(x_{i}(t)-\bar{x}\right)^{2}}
$$

Onde $x_{i}(t)$ corresponde ao valor obtido na amostra e $\bar{x}$ ao valor médio de cada ajuste realizado na máquina durante o teste.

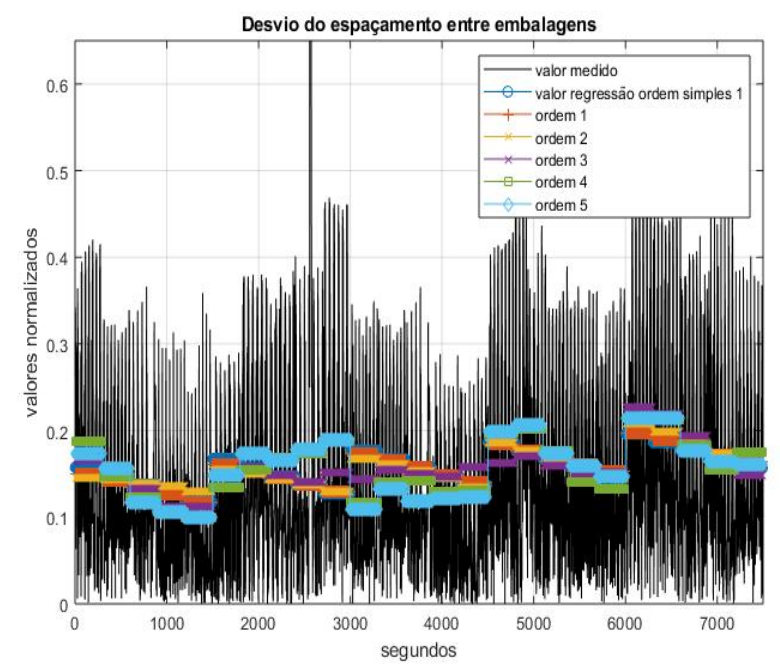

Figura 5. Desvio do espaçamento entre embalagens e os modelos regressores

\section{APRESENTAÇÃO DOS RESULTADOS}

Para interpretar os resultados foram utilizados três indicadores, o coeficiente de determinação $R^{2}$, erro percentural Erro(\%) e o erro médio quadrático $E Q M$. Quanto maior o valor do coeficiente de determinação, melhor é o ajuste para curvas que contenham um mesmo número de amostras, já para os valores de erro, quanto menor, melhor. Esses indicadores foram aplicados nos regressores para a magnitude (Tabela 1) e para o desvio (Tabela 2)

Tabela 1. Indicadores relativos ao espaçamento

\begin{tabular}{cccc} 
Complexidade & $R^{2}$ & $\operatorname{Erro}(\%)$ & $E Q M$ \\
\hline$y_{1}$ & 0.9410 & 6.8272 & 0.0480 \\
$y_{2}$ & 0.9417 & 6.7855 & 0.0477 \\
$y_{3}$ & 0.9461 & 6.5129 & 0.0459 \\
$y_{4}$ & 0.9510 & 6.2082 & 0.0437 \\
$y_{5}$ & 0.9570 & 5.8041 & 0.0410 \\
$y_{6}$ & 0.9606 & 5.5603 & 0.0392 \\
\hline
\end{tabular}

Tabela 2. Indicadores relativos ao desvio do espaçamento

\begin{tabular}{cccc} 
Complexidade & $R^{2}$ & $\operatorname{Erro}(\%)$ & $E Q M$ \\
\hline$y_{1}$ & 0,1808 & 57,2637 & 0,0900 \\
$y_{2}$ & 0,1826 & 57,2020 & 0,0899 \\
$y_{3}$ & 0,1821 & 57,2205 & 0,0899 \\
$y_{4}$ & 0,1948 & 56,7734 & 0,0892 \\
$y_{5}$ & 0,2090 & 56,2694 & 0,0884 \\
$y_{6}$ & 0,2167 & 55,9965 & 0,0880 \\
\hline
\end{tabular}

Observando os dados da Tabela 1 e da Tabela 2, notase que os modelos regressores mais complexos apresentam melhores resultados, pois os mesmos possuem os maiores valores de coeficiente de determinação e simultaneamente apresentam os menores valores nos indicadores de erro, porém não há uma melhoria significativa quando comparados com os resultados apresentados pelos modelos mais simples.

Utilizando o aplicativo curvefittingtool (MathWorks, 2001), foram elaborados mapas de calor para comparar os resultados dos regressores com relação aos ajustes e o valor estimado pela regressão múltipla em relação ao espaçamento das embalagens e os ajustes realizados. A respeito dos mapas de calor gerados, a Figura 6 foi elaborada com base na magnitude do espaçamento entre as embalagens e pode ser comparada com a Figura 7 que representa o regressor mais complexo para esses dados. O mapa de calor da Figura 8 é referente ao desvio calculado para o espaçamento e pode ser comparado com o regressor mais complexo (Figura 9).

Analisando os mapas de calor gerados, as faixas mais escuras correspondem aos menores valores das funções obtidas (espaçamento e desvio), euquanto que as faixas mais claras correspondem aos maiores valores. Também foram calculados a média e o desvio-padrão de cada ajuste realizado na máquina durante o experimento. A Figura 10 mostra a evolução da média durante os ajustes enquando a Figura 11 revela essa mesma evolução para o desviopadrão.

Observando a evolução da média e do desvio-padrão do espaçamento entre embalagens pela Figura 6 e Figura 7 nota-se que nem sempre o ajuste que consegue diminuir o 


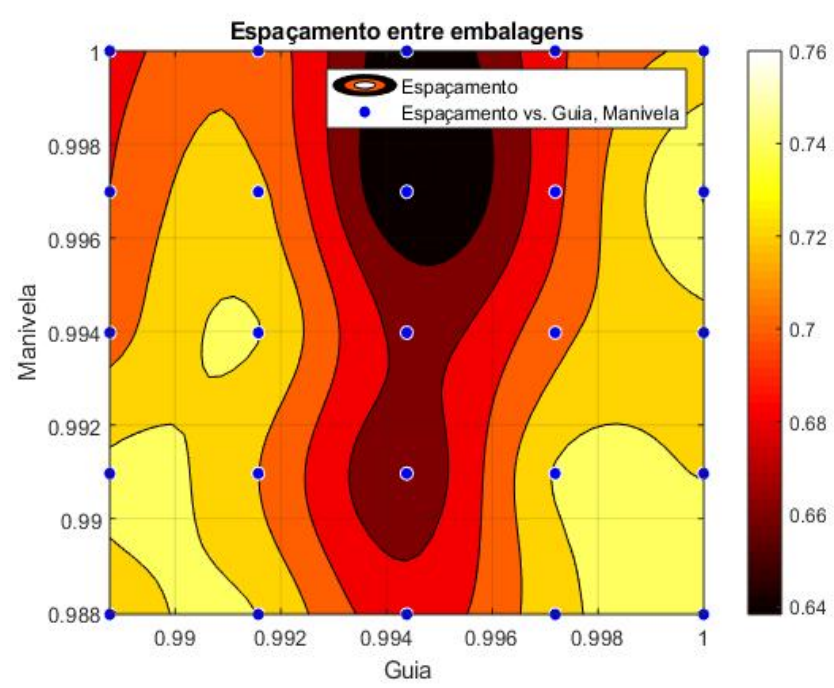

Figura 6. Mapa de calor da magnitude do espaçamento

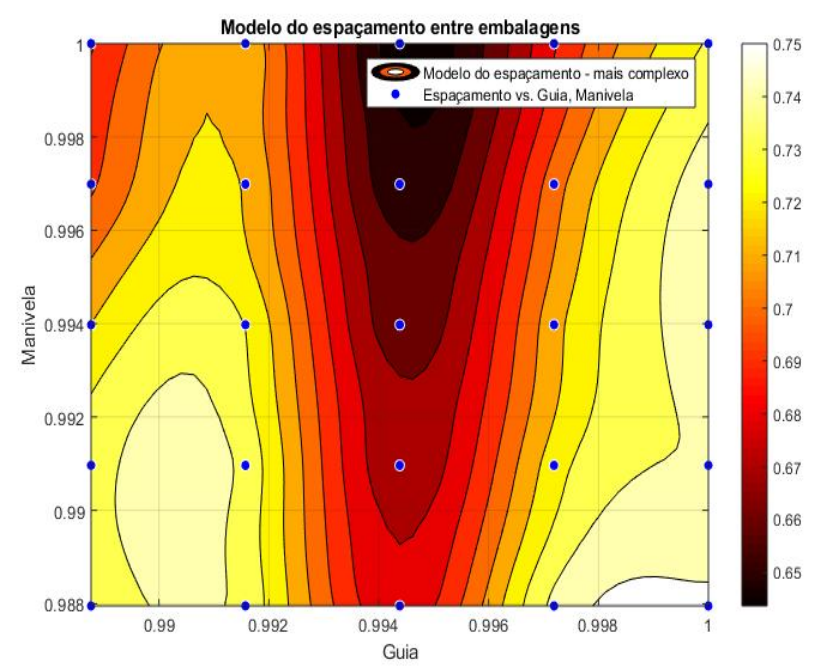

Figura 7. Mapa de calor do regressor mais complexo do valor do espaçamento

valor médio, também diminui o valor de desvio-padrão, ou seja, isso mostra que há uma necessidade de uma esolha onde há de se ponderar o que vale mais a pena na otimização do processo. Para isso é interessante a observação nos mapas de calor gerados na Figura 5 e na Figura 6, pois consegue-se extrair a informação sobre uma possível região de ajustes onde se consiga melhorar, pelo menos um pouco, simultaneamente os valores de magnitude e desvio do espaçamento entre as embalagens no processo de alimentação. Foram gerados mapas de calor (Figura 10) para a evolução dos valores médios em (a) e os valores de desvio-padrão (b) do espaçamento entre as embalagens.

\section{CONCLUSÃO}

Para auxiliar os operadores da máquina em questão, o trabalho que está sendo desenvolvido resultará em análises simples de mapas de calor online, equivalentes aos elaborados neste artigo. Esses mapas podem ser disponibilizados por meio de telas e o operador apenas precisa interpretar

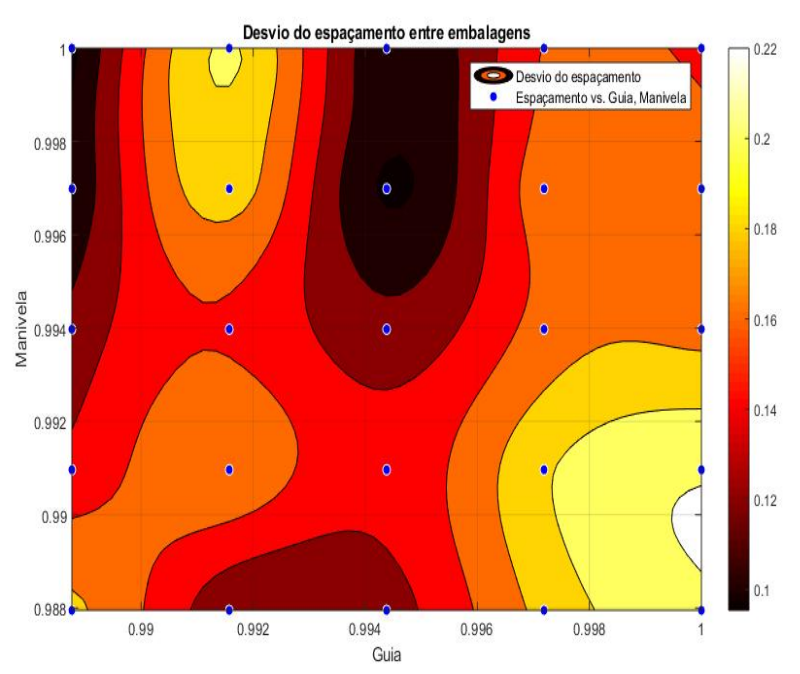

Figura 8. Mapa de calor do desvio do espaçamento entre embalagens

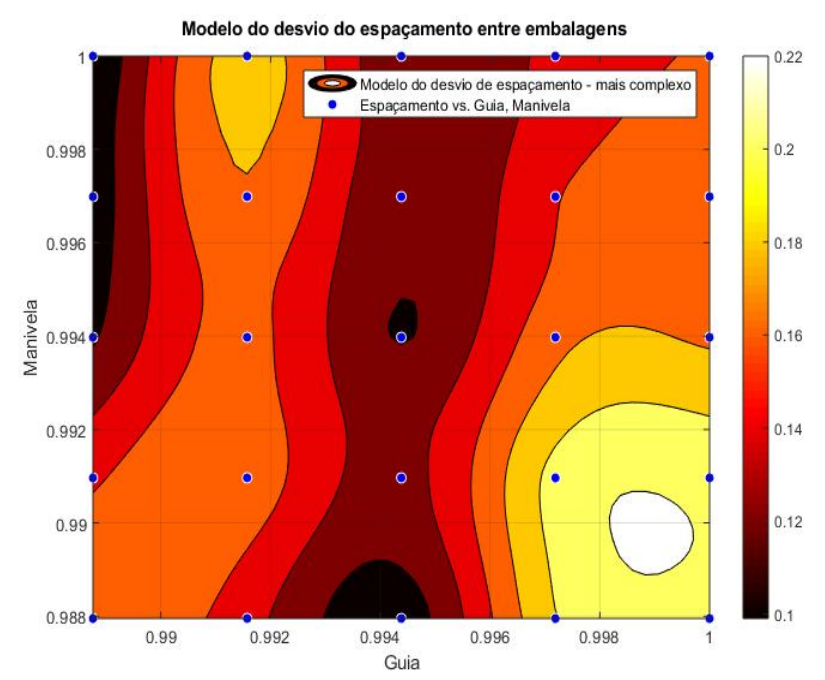

Figura 9. Mapa de calor do regressor mais complexo do valor de desvio do espaçamento

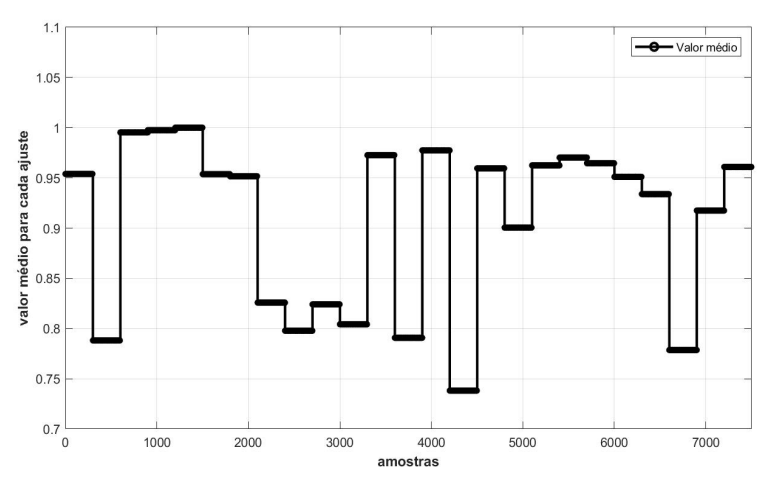

Figura 10. Evolução da média do espaçamento

as cores dos mapas para utilizar uma região de ajustes ótimos para a produção. Vale ressaltar que a identidade da empresa onde está sendo realizado esse trabalho foi ocultada. 


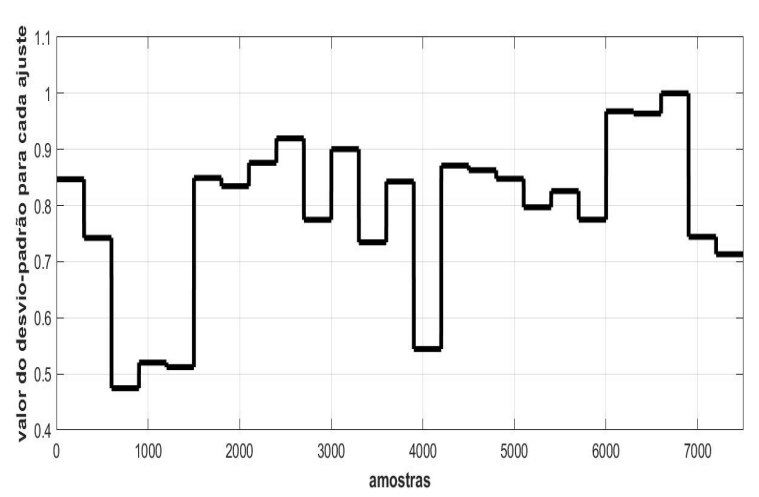

Figura 11. Evolução do desvio-padrão do espaçamento

Pode-se perceber observando os resultados obtidos na Tabela 1 e Tabela 2 que não houve uma variação significativa a respeito dos valores dos indicadores com o aumento da ordem dos polinômios. Os valores referentes ao coeficiente de determinação pouco cresceram, assim como, os valores de erro pouco decaíram.

Quanto aos resultados obtidos na Tabela 1 se apenas for observado os valores numéricos, pode ser que devido a normalização dos dados durante a adaptação dos mesmos, apresentem bons valores porém quando se trabalha com esses dados na prática normalmente não se tem a mesma situação.

Devido a algumas dificuldades como por exemplo, a alta velocidade do processo, conseguir paradas na produção para realizar experimentos, e ao tempo duradouro que pode levar a realização de uma modelagem matemática mais complexa torna-se justificável um estudo de tendência nos processos utilizando ferramentas estatísticas para uma melhoria de controle.

A regressão múltipla mostra uma certa tendência dos valores de espaçamento entre as embalagens com relação aos ajustes mecânicos realizados pelos operadores, porém isso depende da margem de erro que a empresa necessita e essa tendência não atendeu a todos os conjuntos de ajustes.

Vale ressaltar que pode haver comprometimento dos dados por ruído devido ao ambiente industrial em que os sensores estão atuando e uma possível contaminação por outras variáveis não contabilizadas no estudo. Além disso, é importante frisar que cada amostra obtida no experimento é referente ao valor médio de espaçamento de dez embalagens no processo produtivo, ou seja, para se ter uma maior precisão na modelagem dos dados, será aplicado futuramente técnicas de identificação de sistemas mais complexas com o auxílio de equipamentos mais rápidos para a leitura de dados de modo que se tenha os valores de espaçamento entre produtos em tempo real.

Além da utilização de equipamentos melhores, será estudada a exploração de técnicas de otimização multi-objetivo para determinar qual o melhor ajuste, pois observando os dados obtidos pelo sensor verificou-se que nem sempre o comportamento da magnitude e da variação são correspondentes e isso implica em uma escolha que pode ser feita entre ter um sistema mais estável ou um sistema com maior fluxo produtivo médio. A exploração de modelagem dinâmica com aprendizado de máquina também faz parte do escopo das etapas onde as análises mais complexas se farão presentes.

\section{AGRADECIMENTOS}

Os agradecimentos a Deus, as famílias dos autores e a PUCPR pelo incentivo e apoio financeiro.

\section{REFERÊNCIAS}

Hallak, R. and Pereira Filho, A. (2011). Metodologia para análise de desempenho de simulações de sistemas convectivos na região metropolitana de são paulo com o modelo arps: sensibilidade a variações com os esquemas de advecção e assimilação de dados. Revista Brasileira de Meteorologia, 26(4), 591-608.

Lopes, T. and Frota, C. (2015). Aplicação de conceitos do lean manufacturing para melhoria do processo de produção em uma empresa de eletrodomésticos: um estudo de caso. XXXV Encontro Nacional de Engenharia de Produção, Fortaleza, CE, Brasil.

Montgomery, D.C. and Runger, G.C. (2013). Applied statistics and probability for engineers. John Wiley \& Sons, 6th edition.

Moraes, L.H. and Santoro, M.C. (2006). Medida de eficiência em linhas de produção. XXVI ENEGEP.

Moreira, D.A. (2017). Administração da produção e operações. Editora Saraiva.

Provost, F. and Fawcett, T. (2013). Data science and its relationship to big data and data-driven decision making. Big data, 1(1), 51-59.

Robinson, D. (2003). Data reduction and error analysis for the physical sciences. New York: McGraw-Hill.

Rosário, K., Dantas, L., and Oeiras, E. (2015). Aplicação do controle estatístico de processo no monitoramento do peso médio de polpas de frutas: um estudo de caso realizado em uma empresa de médio porte. $X X X V E n$ contro Nacional de Engenharia de Produção. Fortaleza: ENEGEP.

Tessarini, G. and Saltorato, P. (2018). Impactos da indústria 4.0 na organização do trabalho: uma revisão sistemática da literatura. Revista Produção Online, 18(2), 743-769. doi:10.14488/1676-1901.v18i2. 2967. URL https://producaoonline.org.br/rpo/ article/view/2967. 